\title{
Fungos e nematoides associados a sementes de forrageiras tropicais*
}

\author{
Guilherme Mallmann ${ }^{1 * *}$, Jaqueline Rosemeire Verzignassi ${ }^{1}$, Celso Dornelas Fernandes ${ }^{1}$, Jaime Maia dos Santos ${ }^{2}$, \\ Marta Helena Vechiato ${ }^{3}$, Carlos Antonio Inácio ${ }^{4}$, Margareth Vieira Batista ${ }^{1}$, Carolina de Arruda Queiroz ${ }^{1}$
}

${ }^{1}$ Embrapa Gado de Corte. Avenida Rádio Maia, 830, 79002-970, Campo Grande, MS; ${ }^{2}$ Departamento de Fitossanidade, UNESP/FCAV, Via de acesso Prof. Paulo Donato Castellane s/n, 14884-900, Jaboticabal, SP; ${ }^{3}$ Instituto Biológico. Av. Conselheiro Rodrigues Alves, 1252, 04014-140, São Paulo, SP; ${ }^{4}$ Departamento de Fitopatologia, UFRRJ, Rodovia BR 465, Km 7, 23897-000, Seropédica, RJ. * Trabalho financiado pelo CNPq, Fundect e Unipasto. ${ }^{* *}$ Bolsista DCR-CNPq

Autor para correspondência: Guilherme Mallmann (guifito@hotmail.com)

Data de chegada: 04/04/2013. Aceito para publicação em: 23/07/2013.

\section{RESUMO}

Mallmann, G.; Verzignassi, J.R.; Fernandes, C.D.; Santos, J.M.; Vechiato, M.H.; Inácio, C.A.; Batista, M.V.; Queiroz, C.A. Fungos e nematoides associados a sementes de forrageiras tropicais. Summa Phytopathologica, v.39, n.3, p.201-203, 2013.

Objetivou-se avaliar a incidência de fungos e nematoides em sementes de Brachiaria sp. e Panicum maximum produzidas nos estados de Mato Grosso do Sul (MS), Mato Grosso (MT), Goiás (GO), Minas Gerais (MG) e São Paulo (SP). Os principais fungos encontrados nas sementes foram Bipolaris sp., Curvularia sp. e Phoma sp.. As menores incidências destes fungos foram encontradas nas sementes das cultivares BRS Piatã e Xaraés de Brachiaria brizantha e Brachiaria decumbens cv. Basilisk, oriundas dos estados de GO, MG e MS, respectivamente. As cultivares Marandu e BRS Piatã, provenientes das várias regiões, apresentaram elevada ocorrência de Aphelenchoides sp. e Ditylenchus sp.. Sementes da cultivar
Humidicola, de Brachiaria humidicola, produzidas em MS e SP, não apresentaram associação com nematoides. As sementes de Panicum maximum cv. Massai e cv. Mombaça apresentaram maiores incidências de Bipolaris sp., Cladosporium sp., Curvularia sp., Fusarium sp. e Phoma sp., bem como de Aphelenchoides sp. e Ditylenchus sp., especialmente nas sementes produzidas em MT. Alguns dos patógenos encontrados são agentes causais de doenças de grande importância em forrageiras, a exemplo de Bipolaris sp., causando a mancha foliar do Panicum, de alta severidade em Tanzânia, proporcionando sérios comprometimentos da sustentabilidade das pastagens.

Palavras-chave adicionais: Brachiaria brizantha, Panicum maximum, patógeno.

\section{ABSTRACT}

Mallmann, G.; Verzignassi, J.R.; Fernandes, C.D.; Santos, J.M.; Vechiato, M.H.; Inácio, C.A.; Batista, M.V.; Queiroz, C.A. Fungi and nematodes associated with tropical forage seeds. Summa Phytopathologica, v.39, n.3, p.201-203, 2013.

This study aimed to assess the incidence of fungi and nematodes in Brachiaria sp. and Panicum maximum seeds produced in the Brazilian states of Mato Grosso do Sul (MS), Mato Grosso (MT), Goiás (GO), Minas Gerais (MG) and São Paulo (SP). The main fungi found in the seeds were Bipolaris sp., Curvularia sp. and Phoma sp.. The lowest incidence of these fungi was found for seeds of Brachiaria brizantha cultivars BRS Piatã and Xaraés, and Brachiaria decumbens cv. Basilisk, from the states of GO, MG and MS, respectively. The cultivars Marandu and BRS Piatã, from several regions, exhibited high occurrence of Aphelenchoides sp. and Ditylenchus sp.. Seeds of
B. humidicola cultivar Humidicola, produced in MS and SP, did not show association with nematodes. The seeds of Panicum maximum cv. Massai and cv. Mombaça showed higher incidence of Bipolaris sp., Cladosporium sp., Curvularia sp., Fusarium sp. and Phoma sp., as well as Aphelenchoides sp. and Ditylenchus sp., especially for seeds produced in MT. Some of the detected pathogens are causative agents of diseases of major importance in forage plants, such as Bipolaris sp., causing leaf spot in Panicum, of high severity in Tanzânia, which provides serious compromising of the pasture sustainability.

Additional keywords: Brachiaria brizantha, Panicum maximum, pathogen.

Com a expansão das pastagens e intensificação da atividade pecuária nos últimos anos, várias doenças de forrageiras começaram a ter importância significativa, especialmente nas regiões centro-oeste e norte do Brasil, causando perdas em produtividade e qualidade de pastagens.

Pouco se conhece sobre a qualidade sanitária de sementes forrageiras produzidas e comercializadas no país, o que provavelmente contribui para o aumento da incidência de doenças em áreas de pastagens (6).
A associação de patógenos às sementes representa impacto significativo no estande, desenvolvimento das plantas, produção de sementes, persistência das forrageiras no campo e, consequentemente, na produção de leite e carne. Além disso, a presença de patógenos nas sementes constitui entrave para a exportação de sementes $(2,3)$.

Amostras de lotes comerciais de sementes de cultivares Brachiaria sp. e Panicum maximum da safra 2009/2010 e produzidas em diferentes regiões produtoras do país foram analisadas quanto à 
sanidade no Laboratório de Fitopatologia da Embrapa Gado de Corte, em Campo Grande, MS.

Para a detecção dos fungos, utilizou-se o método do papel filtro (Blotter test), sendo analisadas 200 sementes de cada lote, dividindose em dez repetições. As sementes foram incubadas por sete dias em câmara de germinação, à temperatura de $20^{\circ} \mathrm{C}$ e fotoperíodo de 12 horas. Para a extração de fitonematoides foi utilizada a metodologia descrita por Coolen \& D'Herde (1). Para efeito de análise estatística, os valores de incidência de fungos e número de nematoides foram transformados em $(\mathrm{x}+0,01)^{0,5}$. As comparações múltiplas entre as médias foram realizadas pelo teste de agrupamento Scott \& Knott, a $5 \%$ de probabilidade.

Os fungos com maior incidência nas sementes de Brachiaria sp. e Panicum maximum foram Bipolaris sp., Cladosporium sp., Phoma sp., Curvularia sp., Fusarium sp. e Alternaria sp. (Tabela 1). Também foram observadas sementes contaminadas pelos nematoides Aphelenchoides sp. e Ditylenchus sp. (Tabela 2).

$\mathrm{Na}$ cultivar Marandu observou-se elevada incidência de Bipolaris sp., Cladosporium sp. e Curvularia sp. nas sementes produzidas em Mato Grosso. Ainda para esta cultivar, verificou-se incidência de Bipolaris sp., Cladosporium sp., Fusarium sp. e Phoma sp. nas sementes oriundas de Goiás. Os fitonematoides Aphelenchoides sp. e Ditylenchus sp. foram encontrados nas sementes de Marandu nos três estados produtores. Em BRS Piatã e Xaraés, foram identificados os fungos Bipolaris sp., Cladosporium sp., Fusarium sp., Curvularia sp. e Phoma sp., com maior incidência nas sementes oriundas de Mato Grosso do Sul e Mato Grosso. Nas sementes de BRS Piatã, procedentes de Mato Grosso do Sul, Mato Grosso e Minas Gerais, constatou-se a presença de ambos fitonematoides, porém não houve variabilidade na incidência de Aphelenchoides sp. e Ditylenchus sp. em relação aos estados produtores. Em Xaraés, produzida em Goiás, não foi verificada presença de ambos fitonematoides, embora tenha sido confirmada a presença de Aphelenchoides sp. nas sementes oriundas de Mato Grosso do Sul e Mato Grosso, e Ditylenchus sp. foi encontrado apenas nas sementes produzidas em Mato Grosso.

A análise sanitária indicou elevada incidência de fungos nas sementes, com predominância de Bipolaris sp., Cladosporium sp., Curvularia sp., Fusarium sp. e Phoma sp. Estes constituem os fungos fitopatogênicos mais comuns nos campos de produção de sementes de Mombaça nos estados de Mato Grosso do Sul e Mato Grosso. Nas sementes desta cultivar foram encontrados ambos os fitonematoides Aphelenchoides sp. e Ditylenchus sp., porém com menor incidência de Aphelenchoides sp. nas sementes produzidas no estado de Minas Gerais.

Os fungos potencialmente patogênicos e os fitonematoides também foram encontrados em sementes da cultivar Basilisk, de Brachiaria decumbens, procedentes de Mato Grosso e Mato Grosso do Sul. As sementes de P. maximum cv. Massai produzidas em Mato Grosso e São Paulo apresentaram incidência elevada de Cladosporium sp., Curvularia sp., Fusarium sp.. e Phoma sp.. No entanto, não verificaram-se diferenças significativas entre os estados produtores com exceção do fungo Bipolaris sp. Nas sementes da cultivar Massai, provenientes de ambos os estados, foram encontrados os fitonematoides Aphelenchoides sp. e Ditylenchus sp..

Em Humidicola, originária de Mato Grosso do Sul e São Paulo, verificou-se a presença de fungos fitopatogênicos, com ênfase para os gêneros Bipolaris, Curvularia, Fusarium e Phoma, com maior incidência nas sementes de Mato Grosso do Sul. Nesta cultivar não foi verificada a presença de fitonematoides.

Os registros de elevada incidência de fungos observados nas sementes, podem ter como causa as condições climáticas favoráveis nas regiões produtoras. Também a produção de modo continuado na mesma área e/ou em áreas com histórico de ocorrência de fungos, pode favorecer ao aumento da incidência desses patógenos, devido ao incremento do inóculo na área (5).

Apesar das espécies/cultivares avaliadas não terem sido as mesmas

Tabela 1. Incidência de fungos em sementes comerciais de Brachiaria sp. e Panicum maximum em diferentes regiões do Brasil, na safra $2009 / 2010$.

\begin{tabular}{|c|c|c|c|c|c|c|c|}
\hline \multirow{2}{*}{ Espécie } & \multirow{2}{*}{ Estado } & \multicolumn{5}{|c|}{ Incidência de Fungos (\%) } & \multirow[b]{2}{*}{ Phoma sp. } \\
\hline & & Alternaria sp. & Bipolaris sp. & Cladosporium sp. & Curvularia sp. & Fusarium sp. & \\
\hline \multirow[t]{3}{*}{ B. brizantha cv. Marandu } & MS & $0,7^{*} \mathrm{a}$ & $31,7 \mathrm{~b}$ & $23,0 \mathrm{a}$ & $9,5 \mathrm{~b}$ & $7,2 \mathrm{a}$ & $21,2 \mathrm{ab}$ \\
\hline & $\mathrm{MT}$ & $0,5 \mathrm{a}$ & 55,0 a & $24,5 \mathrm{a}$ & $26,2 \mathrm{a}$ & $7,7 \mathrm{a}$ & $10,7 \mathrm{~b}$ \\
\hline & $\mathrm{GO}$ & $0,0 \mathrm{a}$ & $36,0 \mathrm{~b}$ & $19,5 \mathrm{a}$ & $4,0 \mathrm{~b}$ & $12,0 \mathrm{a}$ & $23,0 \mathrm{a}$ \\
\hline \multirow[t]{3}{*}{ B. brizantha cv. Xaraés } & MS & $1,8 \mathrm{a}$ & 66,3 a & 62,0 a & 25,9 a & $10,7 \mathrm{a}$ & 33,1 a \\
\hline & $\mathrm{MT}$ & $1,1 \mathrm{a}$ & 51,2 a & 57,0 a & $16,9 \mathrm{~b}$ & $11,5 \mathrm{a}$ & 33,9 a \\
\hline & $\mathrm{GO}$ & 0,7 a & $24,5 \mathrm{~b}$ & $42,0 \mathrm{~b}$ & $1,7 \mathrm{c}$ & $1,5 \mathrm{~b}$ & $16,0 \mathrm{~b}$ \\
\hline \multirow[t]{3}{*}{ B. brizantha $\mathrm{cv}$. BRS Piatã } & MS & $0,7 \mathrm{a}$ & 48,2 a & 22,3 a & 20,2 a & 7,3 a & $21,2 \mathrm{a}$ \\
\hline & $\mathrm{MT}$ & $0,5 \mathrm{a}$ & $36,7 \mathrm{ab}$ & 18,2 a & $25,5 \mathrm{a}$ & $10,0 \mathrm{a}$ & $26,5 \mathrm{a}$ \\
\hline & MG & $0,0 \mathrm{a}$ & $25,5 \mathrm{~b}$ & $1,0 \mathrm{~b}$ & $5,0 \mathrm{~b}$ & $6,5 \mathrm{a}$ & $11,0 \mathrm{~b}$ \\
\hline \multirow[t]{3}{*}{ P. maximum cv. Mombaça } & MS & $1,5 \mathrm{a}$ & $11,0 \mathrm{a}$ & 59,0 a & $16,5 \mathrm{a}$ & $47,0 \mathrm{a}$ & 36,5 a \\
\hline & $\mathrm{MT}$ & $1,7 \mathrm{a}$ & 20,5 a & 58,5 a & $10,0 \mathrm{a}$ & $13,0 \mathrm{~b}$ & $25,0 \mathrm{a}$ \\
\hline & MG & $0,5 \mathrm{a}$ & $3,5 \mathrm{~b}$ & $0,5 \mathrm{~b}$ & $1,0 \mathrm{~b}$ & $12,5 \mathrm{~b}$ & 27,0 a \\
\hline \multirow[t]{2}{*}{ P. maximum cv. Massai } & $\mathrm{MT}$ & $1,0 \mathrm{a}$ & $24,7 \mathrm{a}$ & $23,0 \mathrm{a}$ & $11,2 \mathrm{a}$ & 5,2 a & 17,5 a \\
\hline & SP & 0,2 a & $4,5 \mathrm{~b}$ & 25,5 a & $7,0 \mathrm{a}$ & $2,7 \mathrm{a}$ & $15,5 \mathrm{a}$ \\
\hline \multirow[t]{2}{*}{ B. decumbens cv. Basilisk } & $\mathrm{MT}$ & $0,7 \mathrm{a}$ & 44,7 a & 16,8 a & 7,8 a & 9,7 a & 20,2 a \\
\hline & MS & 0,1 a & $20,7 \mathrm{~b}$ & 9,1 a & $3,2 \mathrm{~b}$ & $4,2 \mathrm{~b}$ & $7,1 \mathrm{~b}$ \\
\hline \multirow[t]{2}{*}{ B. humidicola cv. Humidicola } & MS & $1,0 \mathrm{a}$ & 29,5 a & 0,0 a & 47,0 a & $5,0 \mathrm{a}$ & $5,5 \mathrm{a}$ \\
\hline & SP & $0,0 \mathrm{a}$ & $7,5 \mathrm{~b}$ & 0,0 a & $23,0 \mathrm{~b}$ & $0,0 \mathrm{~b}$ & $6,0 \mathrm{a}$ \\
\hline
\end{tabular}

*Médias seguidas da mesma letra na coluna, para cada cultivar, não diferem entre si pelo teste de agrupamento Scott Knot $(\mathrm{P} \leq 0,05)$. 
Tabela 2. Número de espécimes de nematoides associados às sementes comerciais de Brachiaria sp. e Panicum maximum em diferentes regiões do Brasil, na safra $2009 / 2010$

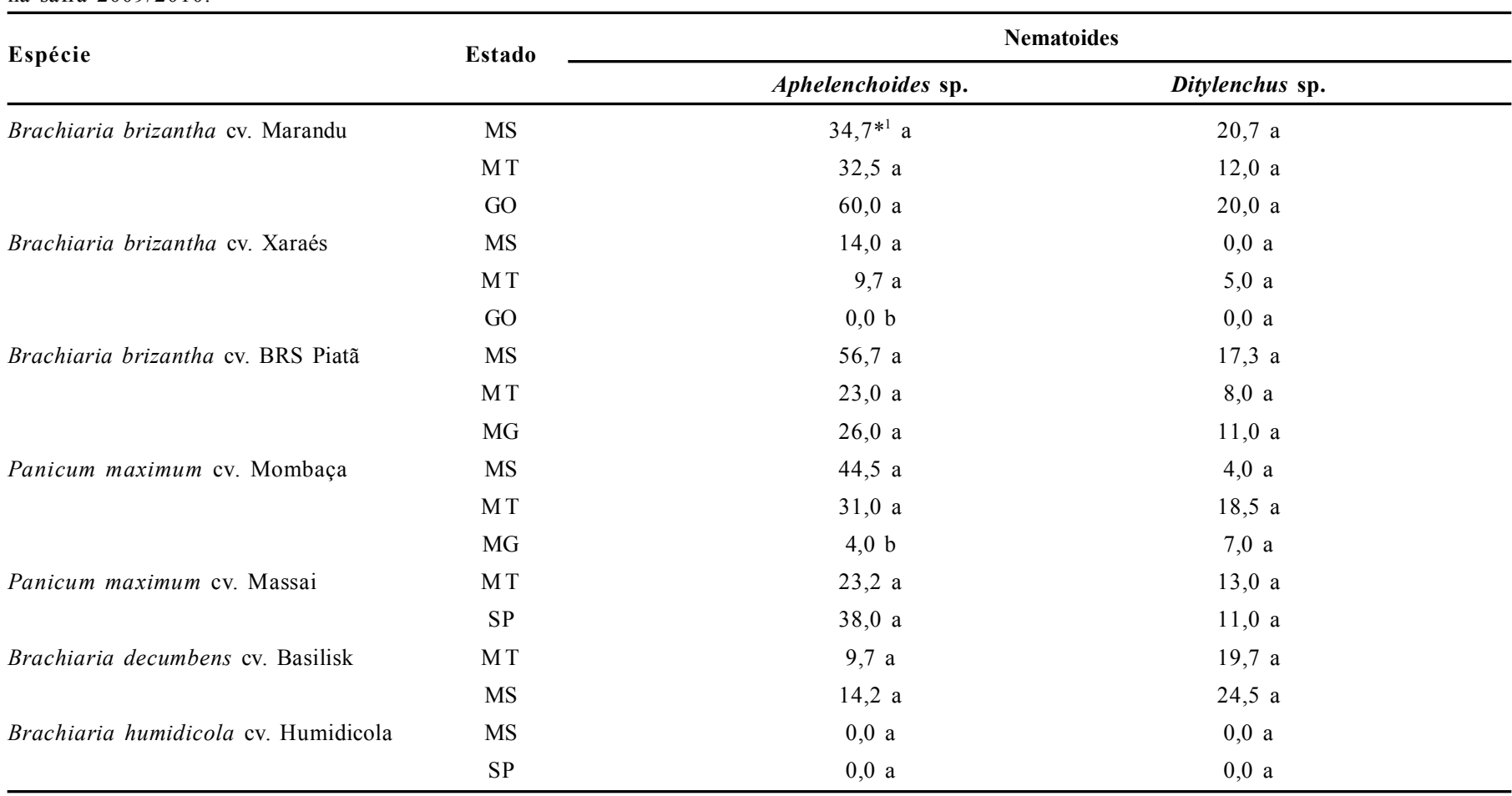

*Médias seguidas da mesma letra na coluna, para cada cultivar, não diferem entre si pelo teste de agrupamento Scott Knot ( $\mathrm{P} \leq 0,05)$. ${ }^{1}$ Número de espécimes por $10 \mathrm{~g}$ de sementes.

em todos os estados, existem diferenças na variabilidade da ocorrência de patógenos em sementes produzidas em diferentes locais, embora todas as regiões apresentem patógenos associados às sementes, afetando negativamente a sua qualidade sanitária.

Os altos níveis de incidência de fungos patogênicos constituíram motivo de preocupação, pois, enquanto alguns fungos são capazes de reduzir a viabilidade das sementes, outros, com crescimento rápido e agressivo como Fusarium sp. e Phoma sp. podem promover a morte da semente antes mesmo da germinação (5).

Acrescenta-se que existem muitos registros acerca da transmissão de fungos patogênicos por meio de sementes, a exemplo de Bipolaris sorokiniana, cuja associação com as sementes constitui a principal fonte de inóculo em cultivos de trigo. Desta forma, o aumento da frequência de Bipolaris sp. nas pastagens (4) poderia estar associado à utilização de lotes de sementes contaminadas. $\mathrm{O}$ fungo tem sido encontrado causando a mancha foliar do Panicum, de alta severidade em Tanzânia, proporcionando sérios comprometimentos da sustentabilidade das pastagens.

Considerando que o Brasil é o maior produtor, consumidor e exportador de sementes de gramíneas forrageiras tropicais do mundo, a comercialização dos lotes também poderá ser prejudicada, principalmente no que diz respeito ao mercado externo, pois existem restrições à presença de alguns fungos e nematoides em sementes por inúmeros países importadores (3).

Em consideração aos resultados apresentados, torna-se incontestável a necessidade de utilização de medidas para evitar a entrada ou que proporcionem redução e/ou eliminação dos patógenos no campo de produção de sementes, estendendo-se até o seu beneficiamento e armazenamento. $\mathrm{O}$ uso de sementes de baixa qualidade sanitária tem sido causa frequente do fracasso na formação de áreas de pastagens, impactando negativamente na sustentabilidade da atividade pecuária.

\section{REFERÊNCIAS BIBLIOGRÁFICAS}

1. Coolen, W.A.; D'Herde, C.J. A method for the quantitative extraction of nematodes from plant tissue. Ghent, 1972. $77 \mathrm{p}$.

2. Fernandes, C.D.; Jerba, V.F.; Verzignassi, J.R. Doenças das plantas forrageiras tropicais. In: Simpósio Brasileiro de Patologia de Sementes, 8., 2004, João Pessoa. Anais. Londrina: Abrates, 2004. p. $51-54$.

3. Fernandes, C.D.; Marchi, C.E.; Jerba, V.F.; Borges, M.F. (2005) Patógenos associados às sementes de forrageiras tropicais e estratégias de controle. In: Zambolim, L. Sementes, qualidade fitossanitária. Viçosa: Universidade Federal de Viçosa, 2005. p.183-213.

4. Marchi, C.E.; Fernandes, C.D.; Jerba, V.F.; Trentin, R.A.; Bueno, M.L.; Guimarães, L.R.; Fabris, L.R. Sementes de forrageiras tropicais: patógenos associados e estratégias de controle. In: Simpósio Brasileiro de Patologia de Sementes, 2006, Passo Fundo. Anais. Londrina: Abrates, 2006. 1 CD-ROM.

5. Menten, J.O.M. Prejuízos causados por patógenos associados às sementes. In: Menten, J.O.M. Patógenos em sementes: detecção, danos e controle químico. São Paulo: Ciba Agro, 1995. p.115136.

6. Vechiato, M.H. Sanidade de gramíneas forrageiras. In: Simpósio Brasileiro de Patologia de Sementes, 8., 2004, João Pessoa. Anais. Londrina: Abrates, 2004. p.55-57. 